\title{
Diagnosis of Dementia in the Specialist Setting: A Comparison Between the Swedish Dementia Registry (SveDem) and the Registry of Dementias of Girona (ReDeGi)
}

\author{
Josep Garre-Olmo ${ }^{\mathrm{a}, \mathrm{b}, 1, *}$, Sara Garcia-Ptacek ${ }^{\mathrm{c}, \mathrm{d}, 1, *}$, Laia Calvó-Perxas ${ }^{\mathrm{a}}$, Oriol Turró-Garriga ${ }^{\mathrm{a}}$, \\ Secundino López-Pousa ${ }^{\mathrm{a}}$ and Maria Eriksdotter ${ }^{\mathrm{c}, \mathrm{d}}$ \\ ${ }^{a}$ Girona Biomedical Research Institute [IDIBGI] - Institut d'Assistència Sanitària, Girona, Catalonia, Spain \\ ${ }^{\mathrm{b}}$ Deparment of Medical Sciences, University of Girona, Girona, Catalonia, Spain \\ ${ }^{\mathrm{c}}$ Department of Neurobiology, Care Sciences and Society, Center for Alzheimer research, Division of Clinical \\ Geriatrics, Karolinska Institutet, Huddinge, Stockholm, Sweden \\ ${ }^{\mathrm{d}}$ Department of Geriatric Medicine, Karolinska University Hospital, Huddinge, Stockholm, Sweden
}

Handling Associate Editor: Martha Dlugaj Jokisch

Accepted 5 May 2016

\begin{abstract}
The aim of this study was to compare the frequency of dementia diagnoses from two dementia registries in Europe. Patients registered between 2007 and 2013 in the Swedish Dementia Registry (SveDem; Sweden) and in the Registry of Dementias of Girona (ReDeGi; North-East of Spain) were selected. We compared sociodemographic data, Mini-Mental State Examination (MMSE) scores, dementia subtype, and medication consumption of 22,384 cases from SveDem and 5,032 cases from ReDeGi. The average age (78.1 years SveDem versus 79.7 years ReDeGi) and the gender (female 58.2\% SveDem versus $61.5 \% \mathrm{ReDeGi}$ ) did not greatly differ. MMSE score at diagnosis was higher for SveDem cases (22.1 versus 17.8). Alzheimer's disease (AD) accounted for the main dementia subtype (36.6\% SveDem versus 55.6\% ReDeGi). The proportion of vascular dementia $(\mathrm{VaD})$ and mixed dementia was higher in SveDem (18.8\% versus $6.4 \%$ and 24.9 versus $13.4 \%$ ), with an odds ratio (OR) and 95\% confidence interval (CI) for SveDem relative to the ReDeGi of 3.41 (3.03-3.84) for VaD, and 2.15 (1.97-2.35) for mixed dementia. This was at the expense of a lower frequency of AD in SveDem (OR 0.41; 95\% CI 0.39-0.44). Other dementia diagnoses such as frontotemporal dementia or dementia with Lewy bodies did not significantly differ between registries $(2.3 \%$ versus $2.9 \% ; 1.9$ versus $3.1 \%)$. Large differences in medication consumption at the time of dementia diagnosis were detected (4.7 treatments SveDem versus 6.8 ReDeGi). Northern and southern European dementia cohorts differ in demographic characteristics, MMSE score at diagnosis, and drug treatment profile.
\end{abstract}

Keywords: Alzheimer's diseae, dementia, epidemiology, registries

\footnotetext{
${ }^{1}$ These authors contributed equally to this work.

${ }^{*}$ Correspondence to: Josep Garre-Olmo, PhD, Edifici Mancomunitat 1, Parc Hospitalari Martí i Julià, C/ Dr Castany s/n; 17190 Salt, Girona (Catalonia) Spain. Tel.: +34 972182600/Ext. 1833; Fax: +34 972189017; E-mail: josep.garre@ias.cat and
}

Sara Garcia-Ptacek, Department of Neurobiology, Care Sciences and Society, Center for Alzheimer research, Division of Clinical Geriatrics, Karolinska Institutet, SE-141 86, Huddinge, Stockholm, Sweden. E-mail: sara.garcia-ptacek@ki.se. 


\section{INTRODUCTION}

Disease registries are a cost-effective way of following patients and obtaining information about a disease process, and are particularly suitable in situations where experimental research is not feasible [1]. Randomized controlled trials require a sturdy working hypothesis and are subject to more ethical concerns: for these reasons a registry may be in a unique position to generate hypotheses to be subsequently confirmed in an experimental trial [1]. Additionally, registries provide information on routine clinical practice, costs, and demographic composition of patient cohorts, all vital to public health planning [2-7].

Demography, prevalence, and survival estimates in dementia vary between regions $[8,9]$. This may be due to underlying differences in lifestyle and geographic factors, differences in health care systems and patient help-seeking behavior, or methodological differences in diagnostic process [9]. Understanding the origin of these differences can provide clues on disease risk factors and set objectives for public health policy. Sweden and Spain belong to the same WHO zone for geography and infant mortality (EURO A) and were grouped for prevalence estimates of dementia in the Delphi consensus, with a prevalence ranging from 1.5 to $24.8 \%$ (depending on age group) for subjects over 65 [8].

Alzheimer's disease (AD) is the most prevalent dementia subtype worldwide $[8,10]$, but the relative impact of different subtypes of dementia presents large regional incidence differences, even within Europe [11-14]. In a multi-center study with eight European countries, including Spain and Sweden, the incidence and prevalence of different dementia diagnoses, particularly of vascular dementia, differed greatly between countries [11, 12]. However, another study did not find differences in the odds of dementia between a composite of Spanish studies and the Kungsholmen study, in Sweden [14]. Prevalence variations are apparent even within countries, for example, in Spain, age- and sex-adjusted prevalence for dementias for +70 populations ranged between $3 \%$ and $12 \%$ depending on the geographical area $[13,15]$. The age-and-sex adjusted prevalence of dementia in Kungsholmen (central Stockholm) was $17.9 \%$ at age 70 in a study from the early 2000 [16]. In Gothenburg, the prevalence of dementia in 70- to 75-year-olds ranged from 1.7 and 6.4\% depending on age group, age cohort, and gender [17]. Since cardiovascular risk factors increase the risk both of $\mathrm{AD}$ and vascular dementia ( $\mathrm{VaD})$, the local incidence of cardiovascular disease might underlie some of these differences [18, 19].

The Swedish Dementia Registry (SveDem) was created in 2007 with the aim of improving quality of care for dementia patients throughout Sweden. Between 2007 and 2013, SveDem registered 35,819 patients with coverage of around $95 \%$ of all specialist memory clinics which diagnose dementia in Sweden [20]. The Registry of Dementia of Girona (ReDeGi) was launched in 2007 and registers demographic and clinical data of all new dementia cases diagnosed at the specialist care level in a delimited geographical area in Catalonia, in the North-East of Spain [5]. Between 2007 and 2013 the ReDeGi registered 5,032 cases.

The aims of this study were to compare the frequency of dementia subtype distribution between two different dementia registries, SveDem and the ReDeGi, and to compare demographics, clinical characteristics, and medication use at time of diagnosis. Comparisons were based in the specialist outpatient diagnoses for both registries.

\section{METHODS}

\section{Design, geographical area of reference, and study population}

We used a cross-sectional design that was based on the data from all cases registered by SveDem and ReDeGi during the years 2007-2013. SveDem's objective is to eventually capture all incident dementia cases in Sweden [2, 20, 21]. Based on incidence estimates, SveDem had coverage of around $31 \%$ of all new dementia diagnoses made nationwide for 2013 (Supplementary Figure 1). However, the coverage of incident dementia diagnoses established at memory clinics is more than $90 \%$ [22]. SveDem operates within the highly decentralized Swedish healthcare system, covering an area of $449,964 \mathrm{~km}^{2}$, a population of over 9.5 million and widely ranging population density with an average of 23.7 inhabitants $/ \mathrm{km}$ [2, 20]. In the Swedish system, both primary care physicians and specialists make diagnoses of dementia, and SveDem registers both. Diagnoses of dementia with early onset and the more rare dementias such as frontotemporal dementia (FTD) and dementia with Lewy bodies (DLB) are usually performed by specialists. The ReDeGi registers all incident cases of dementia diagnosed in the outpatient specialists settings 
belonging to the Health Region of Girona (HRG). Primary care physicians are encouraged to refer suspected dementia cases to specialized care, thus all diagnosis are made in specialist clinics. Diagnoses of dementia may be made in the neurology or geriatrics outpatient consultation offices or in the outpatient memory clinics located in seven public hospitals in the HRG. Based on incidence estimates, the ReDeGi currently has coverage of around $75 \%$ of all new dementia diagnoses made in the HRG [5, 6]. The HRG is located in the northeast region of Catalonia (northeast region of Spain), has an area of $5,517 \mathrm{~km}^{2}$, a population of 746,410 inhabitants, and a population density of 135.3 inhabitants $/ \mathrm{km}^{2}$.

\section{Registry procedure}

In SveDem, newly diagnosed patients meeting ICD-10 criteria for dementia are included in the web-based registry, which provides a framework for recording aspects of diagnostic workup, treatment, care and follow-up [20, 23-25]. The national guidelines for dementia workup established by the Swedish National Board of Health and Welfare [26] are followed in over $85 \%$ of diagnoses, with testing expanded if necessary. Quality control of the database is performed by random cross-checks of histories and entries $[20,25]$. Changing diagnoses within the first year is less than $5 \%$. SveDem is collated with the national population registry to record deaths [23, 25]. At the time of work-up, patients and caregivers are informed orally and in writing about SveDem, can decline participation and withdraw consent at any time. Data is anonymized and analyzed off-site. This study was approved by the Regional ethics committee in Stockholm.

ReDeGi uses standardized criteria for case definition, and follows the guidelines proposed by the Center for Disease Control and Prevention for a surveillance system [27]. The methodological principles and the functional structure of the ReDeGi have been previously described [5]. All diagnoses are based on an interview with the patient and the caregiver, a general medical examination, hematology and blood chemistry tests, and neuroimaging diagnosis if required. A specialist technician of the ReDeGi periodically reviews the medical chart of the cases of dementia notified in each of the seven hospitals of the HRG, and registers demographic and clinical information. The collected information meets the confidentiality requirements for personal data protection in compliance with Spanish legislation.

\section{Variables and data harmonization}

In SveDem, dementia diagnoses are coded as $\mathrm{AD}, \mathrm{VaD}, \mathrm{AD}$ and $\mathrm{VaD}$ (mixed dementia), DLB (McKeith criteria [28]), Parkinson's-disease dementia (PDD, Movement Disorder Society Task Force criteria [29]), FTD (Manchester/Neary criteria [30]), unspecified dementia (where specific dementia diagnosis is not ascertained), and other dementia subtypes (grouping miscellaneous dementia disorders such as corticobasal degeneration or alcohol-related dementias) [25]. Simultaneously, age, gender and baseline Mini-Mental State Examination (MMSE) [31] are entered, as are residency status (living alone versus cohabiting) and place of residence (home versus institution). The number of drugs that the patient takes regularly at the beginning of diagnostic workup is used as a proxy for comorbidity $[2,32]$. The presence or absence of cardiovascular medication, antipsychotics, antidepressants, anxiolytics and hypnotics is recorded. Cholinesterase inhibitors (AChEI) and NMethyl-D-aspartate (NMDA) antagonists prescribed upon diagnosis are entered [25, 30].

In ReDeGi, dementia diagnoses are coded using the DSM-IV-TR diagnostic criteria and additional diagnostic criteria for certain subtypes of dementia (DLB [28], PDD [29], and FTD [30]). Complementary information such as sociodemographic data (age, sex, place of residence, work position, schooling level, marital status, type of housing, healthcare referral device) and clinical data (score and date of administration of the MMSE, the Blessed Dementia Rating Scale score [33] the Clinical Dementia Rating score [34], past family history of dementia, present diagnosis of hypertension, diabetes mellitus, or dyslipidemia, and past history of depressive disorder) is registered. Data of patient's medications are obtained each year by linking the ReDeGi with the database of the Public Catalan Healthcare Service (PCHS), which includes all the drugs prescribed by the PCHS physicians and that have been dispensed in pharmacy offices.

Age, gender, MMSE score, and family history of dementia in first degree relatives were variables directly comparable between registries. In order to harmonize the data, we recorded the following variables: the dementia diagnoses of the ReDeGi were reclassified according to the SveDem classification system; because the categories of the variable residency status did not match between registries, we only used the variable place of residence (home versus institution); since the ReDeGi lacks information 
on the duration of the pharmacological treatments we defined the medications taken regularly by the patients as those drugs with 4 units or more dispensed during one year.

\section{Data analysis}

A descriptive analysis of the variables was performed using central tendency measures and dispersion for quantitative variables. Absolute and relative frequencies were calculated for qualitative variables. Since all cases in ReDeGi are registered in specialist memory clinics, comparisons with SveDem were restricted to specialist diagnoses. The clinical and demographic characteristics of SveDem and the ReDeGi cases were compared using Chisquare tests for categorical variables and Student $t$ tests for continuous variables. In order to quantify the strength of the differences in the distribution of sex, dementia subtype, family history of dementia, and pharmacological treatment between the SveDem and the ReDeGi cases, we calculated odds ratios. We calculated Cohen's $d$ to assess the effect size of the differences in continuous variables such as age and MMSE score between SveDem and the ReDeGi cases. Results are expressed as absolute numbers and percentages, means, standard deviation (SD), and $95 \%$ confidence interval $(95 \% \mathrm{CI})$, as appropriate. Statistical test were considered to be significant with a 2-tailed $p$ value $<0.05$. Data processing and analysis was performed using the Stata S.E. 12.0 for Windows.

\section{RESULTS}

SveDem and the ReDeGi registered 22,384 and 5,032 incident cases of dementia at the outpatient specialist level respectively during the 7-year period. Regarding age and sex, there were no large differences between patients registered in specialist units in SveDem and patients registered in the ReDeGi. Both groups of patients had a mean age around 79 years, and gender distribution was also similar, with a majority of women in both registries, and a 1.5:1 ratio of women-to-men. The percentage of patients reporting family history of dementia in first-degree relatives was higher for SveDem cases (41.6\% versus $26.6 \%$ ). The place of residence differed between the registries; a higher frequency of patients from SveDem compared to patients in the ReDeGi lived in an institution $(8.8 \%$ versus $4.1 \%)$ at the moment of dementia diagnosis. The MMSE mean score between the two registries showed important differences (21.1 points
[SD = 5.1] for SveDem and 17.8 points [SD = 5.4] for the ReDeGi), with a mean difference of 3.2 points $(95 \% \mathrm{CI}=3.0-3.3)$ and a medium effect size (Cohen's $d=0.66$ [95\% CI $=0.63-0.69]$ ). SveDem registered a higher percentage of cases in the upper range level of MMSE score, and patients in the lower range for the MMSE scoring were more frequent in the ReDeGi (Table 1).

The distribution of the different dementia diagnoses showed important differences between the two registries. Specifically, SveDem registered a lower percentage of $\mathrm{AD}$ cases (36.3\% versus $55.6 \%$ ), and a higher number of $\mathrm{VaD}(18.8$ versus $6.4 \%)$ and mixed dementia (24.9 versus $13.4 \%$ ) cases than the ReDeGi. Early-onset AD (EOAD) was registered more frequently in SveDem (3.3\% versus 1.3\%). The $\mathrm{ReDeGi}$ included a higher percentage of patients with other dementia diagnoses (7.2\% versus $2.3 \%$ ) and a lower percentage of patients with unspecified dementia diagnose $(6.7 \%$ versus $10.4 \%)$ than SveDem. Table 2 shows the frequency of dementia diagnosis types for each registry. Supplementary Table 1 reports the dementia diagnoses categories stratified by age groups. When comparing the mean age for the different dementia diagnoses between the two registries it was apparent that, for each diagnostic category, the ReDeGi cases were older than the cases in SveDem. Particularly, these differences showed medium to large effect sizes for AD, DLB, FTD, and PDD (Table 3). Gender distribution across dementia subtypes was similar in both registries, except for the DLB and PDD categories, where men were more frequent in SveDem cases (Table 3). SveDem patients had significantly higher MMSE scores in all types of dementia, and these differences with the ReDeGi showed large effect sizes, irrespective of diagnosis. Excepting EOAD, the frequency of family history of dementia in first-degree relatives was higher in SveDem cases (Table 4).

In SveDem, the mean number of drug treatments at the time of diagnosis was $4.7(\mathrm{SD}=3.3)$, while it was $6.8(\mathrm{SD}=4.1)$ for the ReDeGi (Cohen's $\mathrm{d}=0.61 ; 95 \%$ $\mathrm{CI}=0.57-0.64)$. Regarding the medication subtypes, except for antidepressants, there were important differences between SveDem and the ReDeGi. Overall, the patients registered by SveDem consumed fewer drugs than patients in ReDeGi. The differences ranged from $5.2 \%$ less antipsychotic use to $10.1 \%$ less anxiolytics and sleeping aid use in SveDem compared to the $\mathrm{ReDeGi}$ (Table 5). Regarding AChEI, there was more use in SveDem for AD and mixed dementia, and more use in ReDeGi for other dementia subtypes. 
Table 1

Sociodemographic characteristics of the patients included from 2007 to 2013 in specialist units in SveDem and ReDeGi

\begin{tabular}{lccc}
\hline & $\begin{array}{c}\text { SveDem }^{\dagger} \\
(n=22,384)\end{array}$ & $\begin{array}{c}\text { ReDeGi* }^{*} \\
(n=5,032)\end{array}$ & $\begin{array}{c}\text { Effect size } \\
(95 \% \text { CI })\end{array}$ \\
\hline Age, mean (SD) & $78.10(8.3)$ & $79.72(7.5)$ & $0.19(0.16-0.22)^{\mathrm{a}}$ \\
Sex (female), $n(\%)$ & $13,025(58.2)$ & $3,095(61.5)$ & $0.87(0.81-0.92)^{\mathrm{b}}$ \\
Family history dementia, $n(\%)$ & $6,858(41.6)$ & $1,309(26.6)$ & $1.97(1.83-2.11)^{\mathrm{b}}$ \\
Place of residence, $n(\%)$ & & & \\
$\quad$ Home & $20,398(91.1)$ & $4,654(95.9)$ & $2.27(1.95-2.63)$ \\
$\quad$ Institution & $1,961(8,8)$ & $197(4.1)$ & \\
MMSE, $n(\%)$ & & & \\
$24-30$ & $8,097(36.2)$ & $713(15.2)$ & $3.51(3.22-3.82)^{\mathrm{b}}$ \\
$16-23$ & $10,175(45.5)$ & $2,504(52.0)$ & $0.83(0.78-0.89)^{\mathrm{b}}$ \\
$0-15$ & $3,029(13.5)$ & $1,579(32.8)$ & $0.33(0.31-0.36)^{\mathrm{b}}$ \\
\hline
\end{tabular}

MMSE, Mini-Mental State Examination; ${ }^{\dagger}$ SveDem: 5,914 missing values for family history of dementia; 25 missing values for place of residence; 1,083 missing values for MMSE score; *ReDeGi: 111 missing values for family history of dementia; 181 missing values for place of residence; 236 missing values for MMSE score; 'Cohen's $d$; ${ }^{b}$ Odds ratio of SveDem cohort relative to the ReDeGi.

Table 2

Dementia diagnoses from 2007 to 2013 in SveDem and ReDeGi $[n(\%)]$

\begin{tabular}{lccc}
\hline & $\begin{array}{c}\text { SveDem } \\
(n=22,384)\end{array}$ & $\begin{array}{c}\text { ReDeGi } \\
(n=5,032)\end{array}$ & $\begin{array}{c}\text { Odds ratio } \\
(95 \% \text { CI })\end{array}$ \\
\hline Alzheimer's disease EO & $740(3.3)$ & $68(1.4)$ & $2.49(1.94-3.20)$ \\
Alzheimer's disease LO & $7,396(33.0)$ & $2,728(54.2)$ & $0.41(0.39-0.44)$ \\
Vascular dementia & $4,207(18.8)$ & $319(6.4)$ & $3.41(3.03-3.84)$ \\
Mixed dementia & $5,568(24.9)$ & $670(13.4)$ & $2.15(1.97-2.35)$ \\
Dementia with Lewy bodies & $695(3.1)$ & $243(4.8)$ & $0.63(0.54-0.73)$ \\
Frontotemporal dementia & $515(2.3)$ & $147(2.9)$ & $0.78(0.64-0.94)$ \\
Parkinson's disease dementia & $426(1.9)$ & $158(3.1)$ & $0.59(0.49-0.72)$ \\
Unspecified & $2,330(10.4)$ & $336(6.7)$ & $1.62(1.44-1.82)$ \\
Others & $507(2.3)$ & $363(7.2)$ & $0.29(0.25-0.34)$ \\
\hline
\end{tabular}

EO, early-onset; LO, late-onset. Odds ratios and 95\% confidence intervals (CI) for SveDem patients, relative to ReDeGi, to fall within one of the diagnostic categories.

Table 3

Comparison of age and sex for the different dementia diagnoses in SveDem and ReDeGi

\begin{tabular}{|c|c|c|c|c|c|c|}
\hline & \multicolumn{3}{|c|}{ Age (years, $[$ mean $(\mathrm{SD})]$} & \multicolumn{3}{|c|}{ Sex (female, $[n(\%)])$} \\
\hline & $\begin{array}{c}\text { SveDem } \\
(n=22,384)\end{array}$ & $\begin{array}{c}\text { ReDeGi } \\
(n=5,032)\end{array}$ & $\begin{array}{l}\text { Cohen's } d \\
(95 \% \mathrm{CI})\end{array}$ & $\begin{array}{c}\text { SveDem } \\
(n=22,384)\end{array}$ & $\begin{array}{c}\text { ReDeGi } \\
(n=5,032)\end{array}$ & $\begin{array}{c}\text { Odds ratio } \\
(95 \% \mathrm{CI})\end{array}$ \\
\hline Alzheimer's disease early-onset & $59.4(4.3)$ & $60.5(3.3)$ & $0.26(0.23-0.29)$ & $455(61.5)$ & $48(70.6)$ & $0.67(0.39-1.14)$ \\
\hline Alzheimer's disease late-onset & $78.4(6.6)$ & $81.1(5.6)$ & $0.42(0.39-0.45)$ & $4,839(65.4)$ & $1,840(67.5)$ & $0.91(0.83-1.00)$ \\
\hline Vascular dementia & $79.4(7.6)$ & $77.5(9.1)$ & $0.23(0.20-0.27)$ & $2,082(49.5)$ & $141(44.2)$ & $1.24(0.98-1.55)$ \\
\hline Mixed dementia & $80.9(6.7)$ & $81.2(5.9)$ & $0.04(0.01-0.07)$ & $3,297(59.2)$ & $388(57.9)$ & $1.06(0.89-1.24)$ \\
\hline Dementia with Lewy bodies & $76.6(7.0)$ & $80.6(6.1)$ & $0.58(0.55-0.61)$ & $267(38.4)$ & $137(56.4)$ & $0.48(0.36-0.65)$ \\
\hline Frontotemporal dementia & $69.4(9.3)$ & $74.7(9.4)$ & $0.58(0.53-0.60)$ & $278(54.0)$ & $75(51.0)$ & $1.12(0.78-1.62)$ \\
\hline Parkinson's disease dementia & $74.7(6.9)$ & $79.2(6.2)$ & $0.68(0.64-0.71)$ & $156(36.6)$ & $72(45.6)$ & $0.69(0.47-0.99)$ \\
\hline Unspecified & $78.5(8.9)$ & $81.2(7.4)$ & $0.31(0.28-0.34)$ & $1,414(60.7)$ & $220(65.5)$ & $0.81(0.64-1.03)$ \\
\hline Others & $71.7(10.0)$ & $74.3(11.7)$ & $0.23(0.20-0.26)$ & $237(46.7)$ & $173(47.7)$ & $0.96(0.73-1.26)$ \\
\hline
\end{tabular}

Supplementary Tables 2-9 report the medication at time of diagnosis stratified by dementia subtype.

\section{DISCUSSION}

This study compares the frequency of dementia diagnoses in the specialist setting and patient characteristics between two dementia registries located in the north and south of Europe. Overall, the results show clear differences regarding the frequency subtypes of the dementia diagnoses, cognitive profile, and the medication consumption profile.

All previous epidemiological studies have shown increased prevalence of dementia as age increases, from $1 \%$ in those aged $60-64$ years to $70 \%$ of those aged 90 years and older [11]. The mean age of the 
Table 4

Comparisons of MMSE score and family history of dementia according to dementia group between SveDem and ReDeGi

\begin{tabular}{|c|c|c|c|c|c|c|}
\hline & \multicolumn{3}{|c|}{ MMSE score $[$ mean (SD)] } & \multicolumn{3}{|c|}{ Family history of dementia $([n(\%)])$} \\
\hline & $\begin{array}{l}\text { SveDem }^{\dagger} \\
(n=21,301)\end{array}$ & $\begin{array}{l}\text { ReDeGi* }^{*} \\
(n=4,796)\end{array}$ & $\begin{array}{l}\text { Cohen's } d \\
(95 \% \mathrm{CI})\end{array}$ & $\begin{array}{c}\text { SveDem } \\
(n=16,460)\end{array}$ & $\begin{array}{c}\text { ReDeGi* }^{*} \\
(n=4,921)\end{array}$ & $\begin{array}{c}\text { Odds ratio } \\
(95 \% \mathrm{CI})\end{array}$ \\
\hline Alzheimer's disease early-onset & $22.2(4.9)$ & $19.8(4.8)$ & $0.49(0.46-0.52)$ & $294(48.0)$ & $31(46.3)$ & $1.07(0.64-1.78)$ \\
\hline Alzheimer's disease late-onset & $21.5(5.1)$ & $17.7(5.3)$ & $0.73(0.70-0.77)$ & $2730(47.9)$ & $807(30.2)$ & $2.12(1.92-2.34)$ \\
\hline Vascular dementia & $21.2(4.9)$ & $18.6(5.6)$ & $0.51(0.48-0.54)$ & $972(32.7)$ & $52(16.8)$ & $2.40(1.77-3.27)$ \\
\hline Mixed dementia & $20.9(5.0)$ & $17.1(4.9)$ & $0.76(0.73-0.79)$ & $1738(41.9)$ & $153(23.1)$ & $2.39(1.98-2.90)$ \\
\hline Dementia with Lewy bodies & $21.4(4.9)$ & $17.2(5.5)$ & $0.83(0.80-0.86)$ & $194(38.0)$ & $68(28.7)$ & $1.51(1.08-2.11)$ \\
\hline Frontotemporal dementia & $23.6(5.1)$ & $20.3(6.1)$ & $0.62(0.58-0.65)$ & $147(36.9)$ & $34(23.6)$ & $1.91(1.23-2.95)$ \\
\hline Parkinson's disease dementia & $21.1(4.9)$ & $18.5(5.0)$ & $0.50(0.47-0.53)$ & $94(32.4)$ & $26(16.9)$ & $2.36(1.44-3.84)$ \\
\hline Unspecified & $20.1(5.5)$ & $17.2(5.6)$ & $0.52(0.52-0.55)$ & $597(39.0)$ & $72(22.4)$ & $2.21(1.67-2.93)$ \\
\hline Others & $21.4(5.4)$ & $18.1(5.8)$ & $0.60(0.57-0.63)$ & $92(30.3)$ & $66(18.8)$ & $1.88(1.30-2.70)$ \\
\hline
\end{tabular}

${ }^{\dagger}$ SveDem: 1,083 missing values for MMSE score; 5,914 missing values for family history of dementia; *ReDeGi: 236 missing values for MMSE score; 111 missing values for family history of dementia.

Table 5

Medication (ATC codification) at time of diagnosis $[n(\%)]$

\begin{tabular}{lccc}
\hline & $\begin{array}{c}\text { SveDem }^{\dagger} \\
(n=22,384)\end{array}$ & $\begin{array}{c}\text { ReDeGi* }^{*}(n=4,252) \\
\text { Anticholinesterase (N06DA) }\end{array}$ & $\begin{array}{c}\text { Odds ratio } \\
(95 \% \text { CI })\end{array}$ \\
in AD and mixed dementia & $10,625(48.2)$ & $2,340(55.0)$ & $0.76(0.71-0.81)$ \\
NMDA antagonists (N06DX) & $8,984(66.2 \%)$ & $1,821(60.9 \%)$ & $1.25(1.16-1.36)$ \\
in AD and mixed dementia & $2,534(11.5)$ & $751(17.7)$ & $0.60(0.55-0.66)$ \\
Antidepressants (N06AB) & $1,890(14.0 \%)$ & $611(20.4 \%)$ & $0.63(0.57-0.70)$ \\
Antipsychotics (N05A) & $5,241(25.1)$ & $1,025(24.1)$ & $1.05(0.97-1.13)$ \\
Anxiolytics and/or sleeping & $1,398(6.2)$ & $485(11.4)$ & $0.51(0.46-0.57)$ \\
$\quad$ aids (N05B + N05D) & $4,456(21.4)$ & $1,340(31.5)$ & $0.58(0.54-0.63)$ \\
Cardiovascular (B01AA + B01AC & & & \\
$\quad+$ C02 + C03 + C07 + C08 + C09 + C10) & $14,437(64.5 .0)$ & $3,355(78.9)$ & $0.59(0.54-0.64)$ \\
\hline
\end{tabular}

${ }^{\dagger}$ SveDem: 336 missing cholinesterase inhibitors; 404 missing NMDA; 1,498 missing antidepressants: 1,503 missing antipsychotics; 1,514 missing anxiolytics or sleeping aids; 1,462 missing cardiovascular; ${ }^{*}$ ReDeGi: 780 missing medication profile.

cases in both registries was just under 80 years, with a standard deviation around 8 years, indicating that the great majority of cases in both regions are diagnosed between the age range of 72 and 88 years. According to Eurostat, life expectancy at 65 years for individuals in Sweden and Spain is 20.1 and 21.4 years, respectively, thus, half of the dementia diagnoses are performed in individuals five years before their life expectancy limit, irrespective of the country. This result is in concordance with the findings from the Danish Dementia Registry which includes the secondary health system in the Capital Region of Denmark and which covers 30\% of the Danish population, where the mean age at the time of diagnosis is 78.6 years [35]. Similarly, the French National Alzheimer Database, which covers nationwide secondary care, reports a mean age of 81.9 years for AD cases and 79.3 years for patients with related disorders [36]. Overall, the SveDem and ReDeGi cohorts did not greatly differ in age at diagnosis or gender. However, when stratified by dementia diagnosis, age at onset and gender we detected differences. AD cases in SveDem were slightly younger than those from ReDeGi, while for VaD the pattern was reversed, with ReDeGi cases being younger. DLB and PDD patients in SveDem were younger and more likely to be male than their ReDeGi counterparts. SveDem patients with FTD also tended to be younger with a mean age of 70 years, while the mean for the ReDeGi patients with FTD was around 75 years.

The place of residence at the time of diagnosis is an important variable because it helps estimate the social burden for families living with the patient or the societal economic burden related to the institutionalization [37]. Although more than $90 \%$ of the patients from both registries were living in their own home or with their families, $4.7 \%$ more of Swedish patients were living in an institution at the moment of diagnostic. The French National Alzheimer Database reports $10 \%$ of patients living in nursing homes when they contact the French memory clinics for a suspicion of dementia diagnosis [36]. 
We detect a large difference between registries in the percentage of patients remembering a family history of dementia. Early-onset AD presented differences in frequency between registries, and had the highest frequency of family history of dementia. This result is plausible taking into account that earlyonset AD has been identified as a disease strongly linked with genetic mutations in the amyloid precursor protein gene, in the presenilin 1 gene, and in the presenilin 2 gene [38]. With regard to the rest of dementia categories, the differences may result from variability in the underlying genetic profile of the populations, but also due to a recall bias of the informant or an observer bias of the medical staff during the diagnostic process.

The MMSE score at diagnosis was significantly lower in the ReDeGi cohort, with more patients scoring in the range between 0 to 15 points. These regional differences in cognitive profile have previously been described for $\mathrm{AD}$ [9], and it is interesting to see them replicated for other dementia subtypes (Table 4). Baseline differences in educational attainment may explain these findings [9], especially if we consider the high proportion of cases with low education in the ReDeGi [39]. The Danish registry presents a mean MMSE mean of 20.9 points for all-cause dementia and the French National Alzheimer Database a mean of 16.4 for AD patients, 18.5 for related disorders, and 25.6 for patients with mild cognitive impairment $[35,36]$.

Few previous studies compare dementia cohort composition between different countries in Europe, and those that do most often limit diagnosis to AD and $\mathrm{VaD}[10,31]$. The largest of these included cohorts from eight European countries, among them Sweden and Spain, and found a similar proportion of $\mathrm{VaD}$ $(15-20 \%)$ in all studies, while the prevalence of AD varied more between studies and depended on the percentage of those diagnosed as "other dementias" [11]. In agreement with existing reports, in our study $\mathrm{AD}$ was the most frequently diagnosed dementia in both cohorts, but the proportion of patients receiving a diagnosis of $\mathrm{VaD}$ was three times higher in SveDem than in the ReDeGi (18.8\% versus 6.4\%) as was the mixed dementia diagnoses and these differences were made up by a proportional reduction in AD. This result matches the known North-South gradient in cardiovascular disease [40], which could justify the greater proportion of $\mathrm{VaD}$ in Sweden. Indeed, the incidence for cerebrovascular disease is highest in the north of Europe, decreases to its lowest in the center of Europe (including north of Spain) [40] and then increases again as it reaches the southern coasts. Thus, Catalonia displays one of the lowest incidences of cerebrovascular disease within Europe [41]. Another Spanish study conducted in Valladolid (west of Girona but around the same latitude) found a frequency of $\mathrm{VaD}$ of $5.9 \%$, comparable to the $6.4 \%$ found in the ReDeGi [42]. Interestingly, in a meta-analysis including 48 studies [43], geographical variations of $\mathrm{VaD}$ and $\mathrm{AD}$ were also described in China, where an increased prevalence of $\mathrm{VaD}$ was detected in the North, whereas AD prevalence was found to be similar in the North and in the South. The recent comparison between SveDem and the Danish Dementia Registry also showed a higher frequency of $\mathrm{VaD}$ diagnoses in Sweden $(18.8 \%$ versus $15.3 \%$ ), but due to the similarities between countries, differences were attributed to different tradition for interpretation of diagnostic criteria, better access to brain magnetic resonance imaging (MRI) in Sweden or referral bias, rather than a true difference in disease incidence [35]. A similar pattern was observed for the mixed dementia category, where the proportion was double in SveDem than in the ReDeGi (25\% versus $13 \%$ ). Traditionally the term mixed dementia is used to describe a combination of $\mathrm{AD}$ and $\mathrm{VaD}$, and in clinical settings this diagnostic category is used to classify patients with cognitive dysfunction and impaired functioning in daily life resulting from the coexistence of AD and cerebrovascular pathology, documented either by clinical diagnostic criteria or by neuroimaging findings. Thus, according to the known North-South gradient in cardiovascular disease, it also is plausible to expect a higher number of mixed dementia diagnoses in SveDem than in the ReDeGi. However, differences between countries in the type of specialist that performed the diagnostic (neurologist, geriatrician, psychiatrist), and in the dementia workup, particularly in the high proportion of MRI performed in Sweden, may also be responsible of these findings. Indeed, a higher rate of mixed diagnosis at the expense of $\mathrm{AD}$ was also observed in the comparison of SveDem and the Danish Dementia Registry [35].

Other dementia subtypes are also of interest. DLB in particular is suspected to be underdiagnosed worldwide, with widely ranging estimates from 0 to $23 \%$ of all dementia diagnoses [44, 45]. In SveDem and the $\mathrm{ReDeGi}$, the relative frequencies of DLB diagnosis were not significantly different, and the percentages of 3.1 and 4.8 , respectively, are in line with a percentage of around 4.2 for previous community based studies [44]. PDD's diagnostic frequency 
ranged between $1.9 \%$ in SveDem and $3.1 \%$ in the $\mathrm{ReDeGi}$, and did not differ significantly between registries. These values are close to the $3.6 \%$ of PDD in the general population based on a previous systematic review that included 24 population-based studies to determine the prevalence of this dementia [46]. FTD is believed to occur mainly among individuals under 65 years, and prevalence studies in populations below 65 years have reported low rates $(<1 \%)$ $[47,48]$. The FTD's diagnostic frequency ranged between $2.3 \%$ in SveDem and $2.9 \%$ in the ReDeGi. This result is in concordance with a recent study in a population-based sample of 70-95 year old individuals that reported an overall prevalence between 1.4 and 1.9, depending on the diagnostic criteria used [49]. No significant differences were found between SveDem and the ReDeGi regarding the frequencies of unspecified dementia and other dementias diagnostic categories. The combination of these diagnoses represented $12.7 \%$ and $13.9 \%$ for SveDem and the ReDeGi, respectively.

Concerning the patients' medication profiles at the time of diagnosis, we found an increased consumption of antipsychotics by patients in the ReDeGi compared to those in SveDem. The lower MMSE score at the time of diagnosis may be related to the greater treatment with antipsychotics; however, high rates of antipsychotic consumption have been previously reported both in the ReDeGi $[39,50]$ and in Catalonia [51], which warrants further study regarding mortality risks, medication side-effects, and drug-drug interactions. On the other hand, cardiovascular medications were also more common in the ReDeGi than in SveDem, which seems to be in disagreement with the North-South gradient of cardiovascular risk, but may be related with the differences seen on $\mathrm{AD} / \mathrm{VaD}$ rates.

Some limitations should be considered when interpreting the results hereby presented. First, we lack information on the severity of the behavioral and psychological symptoms of dementia, which would help explaining the differences seen in drug prescription. Second, we do not have information regarding the education level of the registered cases, which may help explaining the differences seen in MMSE scores. Third, although we only considered the cases diagnosed at a specialist level, we cannot rule out a selection bias due to differences in the mechanisms that lead a patient to receive a diagnosis in the two countries. Fourth, we lack information on the length of the treatments and on the dosages prescribed, as well as on over the counter medications.
Fifth, the use of different codification systems for dementia definition (ICD-10 and DSM-IV-TR) may contribute to a classification bias. However, although a discrepancy between results of diagnosing dementia depending on the diagnostic criteria used has been described, the results of a study that compared the concordance of diagnosing dementia using ICD-10 and DSM-IV in daily clinical practice in a large sample of 206 consecutively patients showed a $100 \%$ agreement [52]. Finally, there are systemic differences between care health care settings, and these could influence the time and manner of dementia diagnosis and patient populations. For example, the higher rate of cerebrospinal fluid and MRI in Swedish cohorts could lead to more mixed dementia diagnoses, since vascular lesions are easier to see in MRI and since an $\mathrm{AD}$ component can be detected using cerebrospinal fluid in patients with a more vascular cognitive profile. Furthermore, the greater reach of the Swedish welfare system may lead patients and clinicians to seek early diagnosis in order to receive social support. However, the purpose of this study is to compare naturalistic cohorts, diagnosed following the routine clinical practice in each setting: our cohorts are thus representative of the daily reality of clinical practice in our respective settings. Absent a simultaneous door-to-door survey with homogenized protocols and diagnostic criteria, we will never be able to compare the biological distribution of dementia diagnoses in the North and South of Europe. However, a comparison of real life cohorts represents valuable information to clinicians and policy makers seeking to translate research findings and regulations from different parts of Europe. A key strength of this study is that, regardless the geographical coverage of each registry, both have a valid and reliable method for registering the dementia diagnoses performed in the health system of their countries, allowing comparing profiles and identifying differences of dementia diagnoses between two regions in the northern and southern of Europe. Future collaborations would be of interest to compare pharmacological prescription patterns depending on dementia subtype, the course of the diseases, and mortality rates between registries.

In conclusion, our findings show differences between the profile of patients diagnosed with dementia in a Northern and a Southern European registry. There is a higher percentage of patients with $\mathrm{AD}$ in ReDeGi while VaD and mixed dementia are more frequent in SveDem. Although overall the age at the time of diagnosis is similar, there are differences when comparing dementia diagnoses, 
with ReDeGi cases being older, specifically those with DLB, PDD, and FTD diagnoses. At the time of diagnosis, the mean MMSE score also shows clear differences between countries, with an average difference of 3.2 points higher for SveDem patients. Family history of dementia was $15 \%$ higher in patients from SveDem. The use of anti-dementia and antidepressant treatments at the time of diagnosis does not present large variations between the two registries, however, the use of antipsychotics and anxiolytics or sleeping aids is more frequent in ReDeGi dementia patients. Future longitudinal studies merging these two registries may help to assess how these clinical and demographic features affect the clinical course of the main dementia subtypes.

\section{ACKNOWLEDGMENTS}

The authors thank all patients and caregivers, reporting units, and regional coordinators of SveDem. Thanks to SveDem and the SveDem stirring committee for their support and use of data and figures. Particular thanks to Ann-Katrin Edlund and Karin Westling for their help and knowledge of the SveDem register. The SveDem register is supported by the Swedish Association of Local Authorities and Regions and the Swedish Brain Power network. This study was supported with funds from Stiftelsen för Gamla Tjänarinnor.

The ReDeGi register is supported by the Catalan Health Service - Health Region of Girona. The ReDeGi thank the support and collaboration of the health professionals from the public hospitals actively involved in the register (Department of Neurology, Hospital Universitari Josep Trueta: David Genís, MD; Jordi Gich, PhD; Rosa Meléndez, MD; Albert Molins, MD; LluísRamió, PhD; Joaquín Serena, PhD; Yolanda Silva, PhD; Dementia Unit, Hospital Santa Caterina: Marta Hernández, BPsych; SaioaLejarreta, MD; Jordi Llinàs, BPsych; Manuela Lozano, BPsych; ImmaculadaPericot, PhD; Joan Vilalta-Franch, PhD; Josefa Turbau, MD; Antoni Turon, PhD; Dementia Unit, Hospital de Palamós: ElisabetAlsina, Rosa de Eugenio, MD; Margarita Flaqué, BPsych; Erélido Hernández, MD; Neurology Department, Hospital de Figueres: Olga Carmona, MD; Marta Cullell, BPsych; Teresa Osuna, MD; María del Mar Fernández, MD; Neurology and Geriatrics Departments, Hospital d'Olot: Josep Bisbe, MD; Marta Linares, BPsych; Favià Marquez, MD; Natalia Vallmajó, MD; Neurology and Geriatrics
Departments, Hospital de Blanes: Fernando Espada, MD; Teresa Casadevall, MD; HéctorPerkal, MD; Marta Viñas, MD; Neurology Department; Hospital de Campdevànol: Isabel Casas, BPsych; Anna Manzano, MD; Josep $\mathrm{M}^{\mathrm{a}}$ Cuy, MD, Josefa Turbau, MD).

Authors' disclosures available online (http://jalz.com/manuscript-disclosures/16-0098r2).

\section{SUPPLEMENTARY MATERIAL}

The supplementary material is available in the electronic version of this article: http://dx.doi.org/ 10.3233/JAD-160098.

\section{REFERENCES}

[1] Gliklich RE, Dreyer DN (2010) Registries for Evaluating Patient Outcomes: A User's Guide. (Prepared by Outcome DEcIDE Center [Outcome Sciences, Inc. d/b/a Outcome] under Contract No. HHSA29020050035I TO3.) AHRQ Publication No.10-EHC049. Agency for Healthcare Research and Quality. Rockville, MD.

[2] Johnell K, Religa D, Eriksdotter M (2013) Differences in drug therapy between dementia disorders in the Swedish Dementia Registry: A nationwide study of over 7,000 patients. Dement Geriatr Cogn Disord 35, 239-248.

[3] Religa D, Spangberg K, Wimo A, Edlund AK, Winblad B, Eriksdotter-Jonhagen M (2012) Dementia diagnosis differs in men and women and depends on age and dementia severity: Data from SveDem, the Swedish Dementia Quality Registry. Dement Geriatr Cogn Disord 33, 90-95.

[4] Wimo A, Religa D, Spangberg K, Edlund AK, Winblad B, Eriksdotter M (2013) Costs of diagnosing dementia: Results from SveDem, the Swedish Dementia Registry. Int J Geriatr Psychiatry 28, 1039-1044.

[5] Garre-Olmo J, Flaque M, Gich J, Pulido TO, Turbau J, Vallmajo N, Viñas M, López-Pousa S, Registry of Dementia of Girona Study Group (ReDeGi Group) (2009) A clinical registry of dementia based on the principle of epidemiological surveillance. BMC Neurol 9,5 .

[6] Calvo-Perxas L, Osuna MT, Gich J, Eligio-Hernandez E, Linares M, Vinas M, Casas I, Turro-Garriga O, Lopez-Pousa S, Garre-Olmo J (2012) Clinical and demographic characteristics of the cases of dementia diagnosed in the Health District of Girona throughout the period 2007-2010: Data from the Girona Dementia Registry (ReDeGi). Rev Neurol 54, 399-406.

[7] Calvo-Perxas L, Lopez-Pousa S, Turro-Garriga O, de Eugenio R, Linares M, Fernández Mdel M, Castellanos M, Casas I, Turón-Estrada A, Casadevall T, Coromina J, VilaltaFranch J, Garre-Olmo J (2013) Pain treatment and its cost in old people with dementia: A descriptive analysis from the Registry of Dementias of Girona (ReDeGi). Int J Neurosci 123, 339-346.

[8] Ferri CP, Prince M, Brayne C, Brodaty H, Fratiglioni L, Ganguli M, Hall K, Hasegawa K, Hendrie H, Huang Y, Jorm A, Mathers C, Menezes PR, Rimmer E, Scazufca M, Alzheimer's Disease International (2005) Global prevalence of dementia: A Delphi consensus study. Lancet 366, 2112-2117. 
[9] Hausner L, Frolich L, Gardette V, Reynish E, Ousset PJ, Andrieu S, Vellas B, On Behalf Of The Ictus-Eadc Study Group (2010) Regional variation on the presentation of Alzheimer's disease patients in memory clinics within Europe: Data from the ICTUS study. J Alzheimers Dis 21, 155-165.

[10] Alzheimer's Association (2015) Alzheimer's disease facts and figures. Alzheimers Dement 11, 332-384.

[11] Fratiglioni L, Launer LJ, Andersen K, Breteler MM, Copeland JR, Dartigues JF, Lobo A, Martinez-Lage J, Soininen H, Hofman A (2000) Incidence of dementia and major subtypes in Europe: A collaborative study of populationbased cohorts. Neurologic Diseases in the Elderly Research Group. Neurology 54, S10-S15.

[12] Lobo A, Launer LJ, Fratiglioni L, Andersen K, Di Carlo A, Breteler MM, Copeland JR, Dartigues JF, Jagger C, Martinez-Lage J, Soininen H, Hofman A (2000) Prevalence of dementia and major subtypes in Europe: A collaborative study of population-based cohorts. Neurologic Diseases in the Elderly Research Group. Neurology 54, S4-S9.

[13] de Pedro-Cuesta J, Virues-Ortega J, Vega S, Seijo-Martínez M, Saz P, Rodríguez F, Rodríguez-Laso A, Reñé R, de las Heras SP, Mateos R, Martínez-Martín P, Manubens JM, Mahillo-Fernandez I, López-Pousa S, Lobo A, Reglà JL, Gascón J, García FJ, Fernàndez-Martínez M, Boix R, Bermejo-Pareja F, Bergareche A, Benito-León J, de Arce A, del Barrio JL (2009) Prevalence of dementia and major dementia subtypes in Spanish populations: A reanalysis of dementia prevalence surveys, 1990-2008. BMC Neurol 9, 55 .

[14] Virues-Ortega J, de Pedro-Cuesta J, Vega S, Seijo-Martínez M, Saz P, Rodríguez F, Rodríguez-Laso A, Reñé R, de Las Heras SP, Mateos R, Martínez-Martín P, Mahillo-Fernández I, López-Pousa S, Lobo A, Reglá JL, Gascón J, García FJ, Fernández-Martínez M, Boix R, Bermejo-Pareja F, Bergareche A, Sánchez-SánchezF, de Arce A, del Barrio JL, Spanish Epidemiological Studies on Ageing Group (2011) Prevalence and European comparison of dementia in $\mathrm{a} \geq 75$ year-old composite population in Spain. Acta Neurol Scand 123, 316-324.

[15] Lobo A, Saz P, Marcos G, Dia JL, De-la-Camara C, Ventura T, Montañes JA, Lobo-Escolar A, Aznar S, ZARADEMP Workgroup (2007) Prevalence of dementia in a southern European population in two different time periods: The ZARADEMP Project. Acta Psychiatr Scand 116, 299-307.

[16] Qiu C, von Strauss E, Backman L, Winblad B, Fratiglioni L (2013) Twenty-year changes in dementia occurrence suggest decreasing incidence in central Stockholm, Sweden. Neurology 80, 1888-1894.

[17] Wiberg P, Waern M, Billstedt E, Ostling S, Skoog I (2013) Secular trends in the prevalence of dementia and depression in Swedish septuagenarians 1976-2006. Psychol Med 43, 2627-2634.

[18] Kling MA, Trojanowski JQ, Wolk DA, Lee VM, Arnold SE (2013) Vascular disease and dementias: Paradigm shifts to drive research in new directions. Alzheimers Dement 9 , 76-92.

[19] Muller-Nordhorn J, Binting S, Roll S, Willich SN (2008) An update on regional variation in cardiovascular mortality within Europe. Eur Heart J 29, 1316-1326.

[20] Religa D, Fereshtehnejad SM, Cermakova P, Edlund AK, Garcia-Ptacek S, Granqvist N, Hallbäck A, Kåwe K, Farahmand B, Kilander L, Mattsson UB, Nägga K, Nordström P, Wijk H, Wimo A, Winblad B, Eriksdotter M (2015) SveDem, the Swedish Dementia Registry - a tool for improving the quality of diagnostics, treatment and care of dementia patients in clinical practice. PLoS One 10, e0116538.

[21] Fereshtehnejad SM, Johnell K, Eriksdotter M (2014) Antidementia drugs and co-medication among patients with Alzheimer's disease: Investigating real-world drug use in clinical practice using the Swedish Dementia Quality Registry (SveDem). Drugs Aging 31, 215-224.

[22] SveDem styrgruppen. Årsrapport SveDem 2013 (2014) (SveDem steering committee. Yearly report on SveDem 2013), http://www.ucr.uu.se/svedem/index.php/omsvedem/arsrapporter, Last updated September 22, 2015, Accessed on Septembe 25, 2014.

[23] Garcia-Ptacek S, Kareholt I, Farahmand B, Cuadrado ML, Religa D, Eriksdotter M (2014) Body-mass index and mortality in incident dementia: A cohort study on 11,398 patients from SveDem, the Swedish Dementia Registry. $J$ Am Med Dir Assoc 15, 441-447.

[24] Faxen-Irving G, Fereshtehnejad SM, Falahati F, Cedergren L, Göranzon H, Wallman K, García-Ptacek S, Eriksdotter M, Religa D (2014) Body mass index in different dementia disorders: Results from the Swedish Dementia Quality Registry (SveDem). Dement Geriatr Cogn Dis Extra 4, 65-75.

[25] Garcia-Ptacek S, Farahmand B, Kareholt I, Religa D, Cuadrado ML, Eriksdotter M (2014) Mortality risk after dementia diagnosis by dementia type and underlying factors: A cohort of 15,209 patients based on the Swedish Dementia Registry. J Alzheimers Dis 41, 467-477.

[26] Socialstyrelsen. Nationella riktlinjer för vård och omsorg vid demenssjukdom 2010-stöd för styrning och ledning (2010) (Swedish National Board of Health and Welfare. National Guidelines for Care in Cases of Dementia), https://www.socialstyrelsen.se/Lists/Artikelkatalog/ Attachments/18012/2010-5-1.pdf, Last updated May 1, 2010, Accessed on September 25, 2013.

[27] German RR, Janes GR, Romaguera RA (2001) Lessons learned from the first funding period of the CDC Assessment Initiative. J Public Health Manag Pract 7, 50-57.

[28] McKeith IG (2006) Consensus guidelines for the clinical and pathologic diagnosis of dementia with Lewy bodies (DLB): Report of the Consortium on DLB International Workshop. J Alzheimers Dis 9, 417-423.

[29] Emre M, Aarsland D, Brown R, Burn DJ, Duyckaerts C, Mizuno Y, Broe GA, Cummings J, Dickson DW, Gauthier S, Goldman J, Goetz C, Korczyn A, Lees A, Levy R, Litvan I, McKeith I, Olanow W, Poewe W, Quinn N, Sampaio C, Tolosa E, Dubois B (2007) Clinical diagnostic criteria for dementia associated with Parkinson's disease. Mov Disord 22, 1689-1707.

[30] The Lund and Manchester Groups (1994) Clinical and neuropathological criteria for frontotemporal dementia. $J$ Neurol Neurosurg Psychiatry 57, 416-418.

[31] Folstein MF, Folstein SE, McHugh PR (1975) "Mini-mental state": A practical method for grading the cognitive state of patients for the clinician. J Psychiatr Res 12, 189-198.

[32] Schneeweiss S, Seeger JD, Maclure M, Wang PS, Avorn J, Glynn RJ (2001) Performance of comorbidity scores to control for confounding in epidemiologic studies using claims data. Am J Epidemiol 154, 854-864.

[33] Blessed G, Tomlinson BE, Roth M (1968) The association between quantitative measures of dementia and of senile change in the cerebral grey matter of elderly subjects. $\mathrm{Br} J$ Psychiatry 114, 797-811.

[34] Morris JC (1993) The Clinical Dementia Rating (CDR): Current version and scoring rules. Neurology 43, 24122414. 
[35] Fereshtehnejad SM, Johannsen P, Waldemar G, Eriksdotter M (2015) Dementia diagnosis, treatment, and care in specialist clinics in two Scandinavian countries: A data comparison between the Swedish Dementia Registry (SveDem) and the Danish Dementia Registry. J Alzheimers Dis 48, 229-239.

[36] Anthony S, Pradier C, Chevrier R, Festraets J, Tifratene K, Robert P (2014) The French National Alzheimer database: A fast growing database for researchers and clinicians. Dement Geriatr Cogn Disord 38, 271-280.

[37] Luppa M, Luck T, Brahler E, Konig HH, Riedel-Heller SG (2008) Prediction of institutionalisation in dementia. A systematic review. Dement Geriatr Cogn Disord 26, 65-78.

[38] Piaceri I, Nacmias B, Sorbi S (2013) Genetics of familial and sporadic Alzheimer's disease. Front Biosci (Elite Ed) 5, 167-177.

[39] Calvo-Perxas L, Turro-Garriga O, Aguirregomozcorta M, Bisbe J, Hernández E, López-Pousa S, Manzano A, Palacios M, Pericot-Nierga I, Perkal H, Ramió L, VilaltaFranch J, Garre-Olmo J, Registry of Dementias of Girona Study Group (2014) Psychotropic drugs in patients with Alzheimer's Disease: A longitudinal study by the Registry of Dementias of Girona (ReDeGi) in Catalonia, Spain. J Am Med Dir Assoc 15, 497-503.

[40] Muller-Nordhorn J, Binting S, Roll S, Willich SN (2008) An update on regional variation in cardiovascular mortality within Europe. Eur Heart J 29, 1316-1326.

[41] Marrugat J, Arboix A, Garcia-Eroles L, Salas T, Vila J, Castell C, Tresserras R, Elosua R (2007) The estimated incidence and case fatality rate of ischemic and hemorrhagic cerebrovascular disease in 2002 in Catalonia. Rev Esp Cardiol 60, 573-580.

[42] Tola-Arribas MA, Yugueros MI, Garea MJ, OrtegaValín F, Cerón-Fernández A, Fernández-Malvido B, San José-Gallegos A, González-Touya M, Botrán-Velicia A, Iglesias-Rodríguez V, Díaz-Gómez B (2013) Prevalence of dementia and subtypes in Valladolid, Northwestern Spain: The DEMINVALL study. PLoS One 8, e77688.

[43] Zhang Y, Xu Y, Nie H, Wu Y, Zhang L, Zhang M (2012) Prevalence of dementia and major dementia subtypes in the
Chinese populations: A meta-analysis of dementia prevalence surveys, 1980-2010. J Clin Neurosci 19, 1333-1337.

[44] Vann Jones SA, O'Brien JT (2014) The prevalence and incidence of dementia with Lewy bodies: A systematic review of population and clinical studies. Psychol Med 44, 684.

[45] Stevens T, Livingston G, Kitchen G, Manela M, Walker Z, Katona C (2002) Islington study of dementia subtypes in the community. Br J Psychiatry 180, 270-276.

[46] Aarsland D, Zaccai J, Brayne C (2005) A systematic review of prevalence studies of dementia in Parkinson's disease. Mov Disord 20, 1255-1263.

[47] Gascon-Bayarri J, Rene R, Del Barrio JL, De PedroCuesta J, Ramón JM, Manubens JM, Sánchez C, Hernández M, Estela J, Juncadella M, Rubio FR (2007) Prevalence of dementia subtypes in El Prat de Llobregat, Catalonia, Spain: The PRATICON study. Neuroepidemiology 28, 224-234.

[48] Wada-Isoe K, Uemura Y, Suto Y, Doi K, Imamura K, Hayashi A, Kitayama M, Watanabe Y, Adachi Y, Nakashima K (2009) Prevalence of dementia in the rural island town of Ama-cho, Japan. Neuroepidemiology 32, 101-106.

[49] Gislason TB, Ostling S, Borjesson-Hanson A, Sjögren M, Simoni M, Pantoni L, Skoog I (2015) Effect of diagnostic criteria on prevalence of frontotemporal dementia in the elderly. Alzheimers Dement 11, 425-433.

[50] Calvo-Perxas L, de Eugenio RM, Marquez-Daniel F, Martínez R, Serena J, Turbau J, Vilalta-Franch J, Viñas M, Turró-Garriga O, Roig AM, López-Pousa S, Garre-Olmo J (2012) Profile and variables related to antipsychotic consumption according to dementia subtypes. Int Psychogeriatr 24, 940-947.

[51] Fort I, Formiga F, Robles MJ, Regalado P, Rodriguez D, Barranco E (2010) High prevalence of neuroleptic drug use in elderly people with dementia. Med Clin (Barc) 134, 101106.

[52] Naik M, Nygaard HA (2008) Diagnosing dementia - ICD10 not so bad after all: A comparison between dementia criteria according to DSM-IV and ICD-10. Int J Geriatr Psychiatry 23, 279-282. 